\title{
Brain Transcriptome Sequencing of a Natural Model of Alzheimer's Disease
}

\author{
Francisco Altimiras ${ }^{1,2 * \dagger}$, Barbara Uszczynska-Ratajczak ${ }^{3,4 \dagger}$, Francisco Camara ${ }^{3,4}$, \\ Anna Vlasova ${ }^{3,4}$, Emilio Palumbo ${ }^{3,4}$, Stephen Newhouse ${ }^{5}$, Robert M. J. Deacon ${ }^{6,7}$, \\ Leandro A. E. Farias ${ }^{1}$, Michael J. Hurley ${ }^{8}$, David E. Loyola ${ }^{9}$, Rodrigo A. Vásquez ${ }^{10}$, \\ Richard Dobson ${ }^{5}$, Roderic Guigó ${ }^{3,4 *}$ and Patricia Cogram ${ }^{6,7 *}$ \\ ${ }^{1}$ Faculty of Engineering and Sciences, Universidad Adolfo Ibañez, Santiago, Chile, ${ }^{2}$ Telefonica Research and Development, \\ Santiago, Chile, ${ }^{3}$ Centre for Genomic Regulation, Barcelona Institute of Science and Technology, Barcelona, Spain, \\ ${ }^{4}$ Universitat Pompeu Fabra, Barcelona, Spain, ${ }^{5}$ Institute of Psychiatry, Psychology and Neuroscience, King's College \\ London, London, UK, ${ }^{6}$ Laboratory of Molecular Neuropsychiatry, Institute of Cognitive and Translational Neuroscience \\ (INCYT), INECO Foundation, Favaloro University, National Scientific and Technical Research Council, Buenos Aires, \\ Argentina, ${ }^{7} \mathrm{GeN} . D D I$ Ltd, London, UK, ${ }^{8}$ Division of Brain Sciences, Centre for Neuroinflammation and Neurodegeneration, \\ Imperial College, London, UK, ${ }^{9}$ National Center for Genomics and Bioinformatics, Santiago, Chile, ${ }^{10}$ Faculty of Sciences, \\ Institute of Ecology and Biodiversity, Universidad de Chile, Santiago, Chile
}

Keywords: Alzheimer's disease, Octodon degus, RNA-seq, gene expression, animal models

\section{INTRODUCTION}

OPEN ACCESS

Edited by:

George E. Barreto,

Pontifical Xavierian University,

Colombia

Reviewed by:

Ángel M. Carrión,

Pablo de Olavide University, USA Odete A. B. Da Cruz E. Silva, University of Aveiro, Portugal

*Correspondence: Francisco Altimiras fjaltimiras@gmail.com

Roderic Guigó

roderic.guigo@crg.eu

Patricia Cogram

patricia.cogram@gmail.com

${ }^{\dagger}$ Shared first authorship.

Received: 03 November 2016 Accepted: 01 March 2017 Published: 20 March 2017

Citation:

Altimiras F, Uszczynska-Ratajczak B, Camara F, Vlasova A, Palumbo E, Newhouse S, Deacon RMJ,

Farias LAE, Hurley MJ, Loyola DE, Vásquez RA, Dobson R, Guigó $R$ and Cogram P (2017) Brain Transcriptome

Sequencing of a Natural Model of Alzheimer's Disease.

Front. Aging Neurosci. 9:64. doi: 10.3389/fnagi.2017.00064
Alzheimer's disease (AD) is a slowly progressive disease characterized by impairment of memory and eventually by disturbances in reasoning, planning, language, and perception. Ageing is the greatest risk factor for its development but mutations in amyloid precursor protein (APP), apolipoprotein E (APOE), microtubule-associated protein tau (MAPT) among others, are also a major factor (Blasko et al., 2004). The symptoms of AD result from neurofibrillary tangles that are composed of aggregates of hyper-phosphorylated tau protein and an increase in the production of amyloid-beta $(\mathrm{A} \beta)$ protein in the brain that leads to deposits of senile plaques. As such, there is a worldwide effort to find an effective disease-modifying treatment that can reverse symptoms and/or delay onset of the disease. Transgenic mouse models exist that mimic a range of AD-related pathologies, although none of the models fully replicate all pathological features of the human disease (Birch et al., 2014). Drugs developed using these mouse models have failed in phase III clinical trials (Mangialasche et al., 2010; Braidy et al., 2012; Saraceno et al., 2013). These failures question not only our accurate understanding of the disease (Castellani and Perry, 2012) but also the validity of the animal models upon which the drug discovery efforts are rooted (Windisch, 2014; Nazem et al., 2015).

Animal models have contributed significantly to our understanding of the underlying mechanisms of AD. To date, however, these findings have not resulted in target validation in humans and successful translation to disease-modifying therapies. The Octodon degus (O. degus) is a model that naturally integrates multiple AD pathological hallmarks like tau fibrilary tangles and $\beta$-amyloid deposits (Inestrosa et al., 2005, 2015; Deacon et al., 2015). The A $\beta$ peptide sequence in $O$. degus is $97.5 \%$ homologous to the human $A \beta$ peptide sequence (Inestrosa et al., 2005). The species presents acetylcholine (AChE)-rich pyramidal neurons in their forebrain, which decline in numbers during the progression to an $\mathrm{AD}$-like behavioral state, similar to that seen in $\mathrm{AD}$ patients (Ardiles et al., 2012). Affected O. degus also present the characteristic medical signs and symptoms surrounding AD like macular degeneration, diabetes and circadian rhythm dysfunction (Laurijssens et al., 2013). Behavioral experiments have shown that the O. degus can also present behavioral deficits and neural alterations in the frontal cortex and aggression similar to those seen in patients with AD (Tarragon et al., 2013). Most importantly, the O. degus shows a correlation of expression with human $\mathrm{AD}$ - related genes making this model a powerful tool to characterize the effects of novel treatments for AD and identify new therapeutic targets. Our findings advance the use of the $O$. degus as an effective tool for $\mathrm{AD}$ research. 


\section{MATERIALS AND METHODS}

\section{Animals}

In this study O. degus (Rodentia: Octodontidae) were captured from a natural population in central Chile, $30 \mathrm{~km}$ west of Santiago (Rinconada de Maipú, RM). In this environment, degus typically breed once per year in late autumn (May-June), with conceptions in late winter to early spring (September-October). The animals were captured as juveniles during early austral summer (November), when degus are 2-3 months old, with mesh made traps with a Sherman-trap type mechanism. Juveniles corresponded to individuals weighing 70-130 g (females) or 70$140 \mathrm{~g}$ (males), and hence it is possible to differentiate them categorically from adults, which weight above $130 \mathrm{~g}$ for females, $140 \mathrm{~g}$ for males (Ebensperger and Hurtado, 2005; Correa et al., 2016).

They were housed in standard metal cages $50 \times 40 \times 35$ $\mathrm{cm}$ with a layer of wood shaving as bedding and containing a small metallic box $(25 \times 15 \times 10 \mathrm{~cm}$ with a single entrance $)$, under natural photoperiod (12 h light/dark; starting 7 a.m.) and in an air-conditioned animal facility at the Faculty of Sciences, University of Chile (Santiago, Chile). They were fed with a commercial rodent diet (Prolab RMH 3000, Lab diet). Water and food were provided ad libitum during the entire experimental period. Behavioral assessment of a population $(N=84)$ of 3 -years old $O$. degus was performed using the burrowing test in an earlier work (Deacon et al., 2015), to address behavioral dysfunctions in activities of daily living (ADL) (Deacon, 2006, 2014). The population was divided in terms of burrowing performance into two groups: poor-burrowers (PB) and good-burrowers (GB). Brain extract samples were obtained from 8 females (four per each group). Brain soluble $\mathrm{A} \beta_{1-42}$ peptide was determined for these 8 animals by MALDI-TOF MS as described in earlier work (Deacon et al., 2015). Samples were classified according to burrowing performance and brain soluble $A \beta_{1-42}$ levels. Samples from four $\mathrm{PB}$ animals, with an increased level of soluble brain $\mathrm{A} \beta_{1-42}$ (AD-like group), and three samples from $\mathrm{GB}$ animals (with soluble levels of $A \beta_{1-42}$ used as control group) were used for further analysis. Brain tissues were frozen directly in RNAlater solution (Ambion) and stored at $-80^{\circ} \mathrm{C}$ until use. All procedures of capture, transportation, maintenance and experimentation followed the recommendations of the ethics committee of the Faculty of Sciences of the University of Chile, and complied with Chilean regulations (SAG-Chilean Agriculture and Livestock Service) as well as recommendations by the Animal Behavior Society.

\section{Total RNA and mRNA Isolation}

For total RNA extraction, PureLinkTM RNA Mini kit (Ambion, Life Technologies) was used according to the manufacturer's instructions. For genomic DNA digestion, RNase-Free DNase I (Ambion) was used. Total RNA samples were quantified in a Nanodrop 2000 spectrophotometer and integrity was evaluated by agarose gels electrophoresis with a standard protocol. For mRNA isolation from total RNA samples, MicroPoly(A) Purist kit was used according to the manufacturer's instructions. For sizing, quantitation and quality control of
mRNA Bioanalyzer system (Agilent) was used, discarding samples with an RIN $<7$.

\section{RNA-sequencing}

RNA-seq was performed at the National Center for Genomics and Bioinformatics (Santiago, Chile) including library preparation (using SOLiD Total RNA-seq kit), fragmentation and PCR enrichment of target mRNA according to strandspecific protocols. Two batches of paired-end $(75 \times 35 \mathrm{bp})$ library sequencing were done in a SOLiD 5,500 $\times 1$ system (Applied Biosystems).

\section{Gene Prediction}

We combined both $a b$ initio and evidence-based approaches by using the Program to Assemble Spliced Alignments (PASA) and Evidence Modeler (EVM) to de novo annotate protein coding genes in O. degus genome assembly (v1.0) (Haas, 2003). In total 25,621 transcript models were generated by PASA from an initial set of 1,767,640 sequences including (1) 26,240 O. degus mRNA transcripts and (2) 1,741,759 ESTs and mRNA sequences from four species of rodents: mouse, guinea pig, Chinese hamster and rat. Transcript sequences for all five species were derived from the GenBank NCBI databases. We also used $>30$ million UniRef90 protein clusters and 26,259 highly curated rodent-specific SwissProt proteins that were split-mapped to the O. degus genome by using SPALN2 (Iwata and Gotoh, 2012) and EXONERATE (Slater and Birney, 2005) with mouse-specific parameters. The protein-coding gene predictions were obtained on the latest repeat-masked reference assembly of the $O$. degus genome by using the existing mammalian-specific parameter files of four $a b$ initio gene prediction programs: Augustus, Geneid, SGP2, and SNA (Guigó et al., 1992; Guigo et al., 2003; Parra et al., 2003; Stanke et al., 2006a,b). For SGP2, TBLASTX (Altschul, 1997) alignments between human (hg38) and O. degus were additionally used to improve the accuracy of gene predictions (Table S1). We also used external evidence for Geneid including PASA-derived introns, while for Augustus we used both PASAderived intron and exon hints. Performance of all programs with and without external evidence was evaluated for accuracy on an artificial scaffold made up of 238 concatenated $O$. degus transcripts taken from the NCBI reference annotation. The alignments (transcript and protein) and $a b$ initio gene models were combined into consensus CDS models using Evidence Modeler (Haas et al., 2008). The initial gene set was filtered to remove reference gene models supported exclusively by SNAP $a b$ initio predictions. The single-exon consensus gene models derived from predictions solely supported by Geneid, SGP2 or AUGUSTUS with length $<300$ nucleotides and an EVM score $<5$ were also excluded. The weights of each given source were chosen empirically and based on suggestions contained in the EVM documentation. The highest weights were given to transcript alignments, followed by protein alignments and finally $a b$ initio predictions (Augustus, Geneid, SGP2, and SNAP, Table S2). The consensus gene models were loaded into the PASA database and passed through five rounds of annotation updates to add UTRs and alternative splicing variants (Table S3). 


\section{Functional Annotation}

Predicted protein-coding genes in the O. degus genome were further functionally annotated using an in-house developed, automatic pipeline. For each protein sequence we assigned protein signatures, orthology groups, as well as annotated metabolic pathways and reactions using an orthology-based approach. In this analysis we used InterProScan v.5 (Zdobnov and Apweiler, 2001) to scan though all available InterPro databases, including PANTHER, Pfam, TIGRFAM, HAMAP, and SUPERFAMILY and to specify different protein coding signatures in predicted protein coding genes. The protein signatures (protein families, regions, domains, repeats and sites) were further employed to investigate the classification and to assign biological functions to predicted proteins. Blast2GO (Götz et al., 2008) analysis was used to identify GO terms for the predicted proteins, while KEGG Automatic Annotation Server (KAAS) (Moriya et al., 2007) was employed to compare protein sequences against KEGG (Kanehisa et al., 2012, 2014) orthology (KO). In this analysis, KASS applied its bi-directional best hit $(\mathrm{BBH})$ method in homology search against a representative gene set from 33 different species, including Mus musculus and Cricetulus griseus. KO identifiers were then used to retrieve the KEGG relevant functional annotation, such as metabolic pathways and external database references. In addition, we assigned the NCBI gene names from the O. degus reference NCBI annotation (ref_OctDeg1.0) to the predicted genes. This was done by taking NCBI gene names from the corresponding, NCBI annotated proteins, showing full sequence similarity. The sequence similarity was measured by assigning SHA1 checksum to each protein in both proteomes, followed by comparing those sums.

\section{Mapping O. degus Brain RNA-Seq Samples}

The $O$. degus RNA Sequences were aligned to a reference transcriptome obtained from the masked primary genome assembly version 1.0 (WGS Project: AJSA01) and the EVM-based genome annotation. The transcriptome sequence was prepared using RSEM version 1.2.12 (Li and Dewey, 2011) and projected from base space to SOLiD color space using SHRiMP version 2.2.3 (Rumble et al., 2009). The latter program was also employed to align SOLiD $75 \times 35$ bp pair-end sequenced reads to the reference transcriptome according to the following non-default parameters: -h 80\% -o 10 -p opp-in -no-half-paired.

\section{Mapping Human Brain RNA-Seq Samples}

The RNA-seq data set consisting of 6 human brain samples (3 AD subjects and 3 controls, University of Kentucky brain bank) was derived from the National Center for Biotechnology Information Sequence Read Archive database (ncbi.nlm.nih.gov/sra, accession no. SRA060572). Next, pairend reads were mapped to the human reference transcriptome using our in-house pipeline that combines STARv.2.4.0.1 (Dobin et al., 2013) and RSEM version 1.2.12 (Table S4). The reference transcriptome was obtained from human reference genome hg38 and GENCODE v21 (Harrow et al., 2012) as a reference annotation.

\section{Expression Profiling of Human and O. degus Brain Samples}

The number of reads mapped to each gene was calculated using SAMtools (Li et al., 2009). The statistical significance of expression profile for each gene between two groups was determined using edgeR, an open source R/Bioconductor package (Robinson et al., 2010). In this study, to estimate the significance of gene expression difference between $\mathrm{AD}$ or $\mathrm{AD}$ like (PB) subjects and human controls or O. degus (GB) controls, the absolute value of $\log 2$ Ratio $(\log F C \geq 1)$, $\log$ read count per million reads $(\log C P M>1)$ and $\mathrm{FDR}<0.05$ were used as a criterion. Raw gene reads counts were also normalized to RPKM values (reads per kilobase per million mapped reads) using the RPKM formula described by Mortazavi et al. (2008).

\section{GO Enrichment Analysis for Differentially Expressed Genes}

Functional enrichment analysis for $O$. degus was performed using Fisher's exact test implemented in R programming language. The GO enrichment analysis for human was also done using $\mathrm{R}$. The GOstats package was used to detect the significantly enriched GO terms for DEGs, which were compared to the full GENCODE 21 gene set. For both species, we further analyzed top $20 \mathrm{GO}$ terms ranked by $p$-value with $p<0.05$.

\section{RESULTS}

\section{Gene Prediction and Functional Annotation}

With the aim to enrich the current version of $O$. degus genome annotation with the new data obtained in this study, we performed de novo protein coding gene annotation. Our Evidence-Modeler (EVM) based annotation of repeat-mask $O$. degus genome (v1.0) resulted in prediction of 31,739 proteincoding genes, corresponding to 36,866 predicted transcripts and 36,575 proteins. The comparison of EVM-based and NCBI reference annotation revealed that the number of protein coding genes increased by 1.52 -fold from 20,779 (NCBI) to 31,739 (EVM-based). Similar 1.40-fold change was reported for protein coding transcripts (26,248 for NCBI and 36,866 for EVM-based annotation) (Table S3).

Each protein sequence was functionally annotated using the in house automated pipeline. Annotation features were assigned to a total of 35,618 (97\%) proteins, 30,661 (97\%) genes), of these $29,847(82 \%)$ of the proteins were assigned some GO terms (Tables S5, S6, Figures S1, S2). This functional annotation, including GO terms and KEGG orthology groups, allowed us to perform enrichment analysis for genes of interest.

\section{The Brain Transcriptome of AD-like O. degus}

After mapping the reads to the O. degus genome, we performed pairwise comparisons to measure the gene expression level differences between $\mathrm{AD}$-like subjects and controls. As a result 54 DEGs were identified between those two groups. Statistical analysis revealed 29 genes to be up- and 25 to be down-regulated 

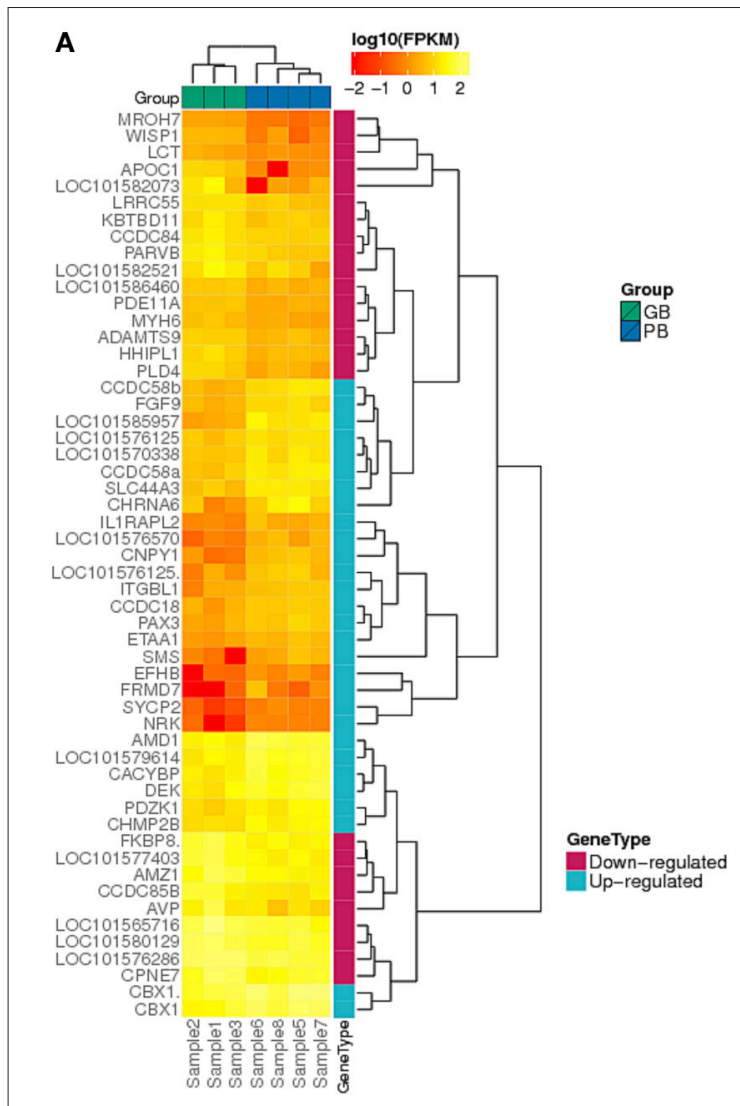

B

\begin{tabular}{|c|c|c|c|}
\hline KEEG PATHWAY & DESCRIPTION & KEEG PATHWAY & DESCRIPTION \\
\hline \multicolumn{2}{|c|}{\begin{tabular}{|c|} 
UP-REGULATED GENES \\
\end{tabular}} & \multicolumn{2}{|c|}{\begin{tabular}{|c|} 
DOWN-REGULATED GENES \\
\end{tabular}} \\
\hline Ko04015 & Rap1 signaling pathway & Ko01100 & Metabolic pathways \\
\hline Ko04111 & Cell cycle - yeast & Ko04260 & Cardiac muscle contraction \\
\hline Ko04810 & Regulation of actin cytoskeleton & Ko00190 & Oxidative phosphorylation \\
\hline Ko04010 & MAPK signaling pathway & K005032 & Morphine addiction \\
\hline Ko04014 & Ras signaling pathway & Ko05010 & Alzheimer's disease \\
\hline Ko05231 & Choline metabolism in cancer & Ko00140 & Steroid hormone biosynthesis \\
\hline Ko04725 & Cholinergic synapse & K004962 & Vasopresin-regulated water reabsorption \\
\hline Ko05218 & Melanoma & K004726 & Serotonergic synapse \\
\hline Ko05200 & Pathways in cancer & K004932 & Non-aclholic fatty liver disease \\
\hline Ko04144 & Endocytosis & Ko00230 & Purine metabolism \\
\hline Ko05033 & Nicotine addiction & Ko04973 & Carbohydrate digestion and absorbtion \\
\hline Ko04080 & Neuroactive ligand-receptor interaction & Ko04510 & Focal adhesion \\
\hline \multirow[t]{9}{*}{ Ko04151 } & PI3K-Akt signaling pathway & Ko05012 & Parkinson's disease \\
\hline & & Ko00052 & Galactose metabolism \\
\hline & & K004261 & Adrenergic signaling in cardiomyocytes \\
\hline & & K005416 & Viral myocarditis \\
\hline & & Ko04530 & Tight junction \\
\hline & & Ko05016 & Huntington's disease \\
\hline & & Ko03010 & Ribosome \\
\hline & & Ko00564 & Glycerophospholipid metabolism \\
\hline & & Ko00565 & Ether lipid metabolism \\
\hline
\end{tabular}

C
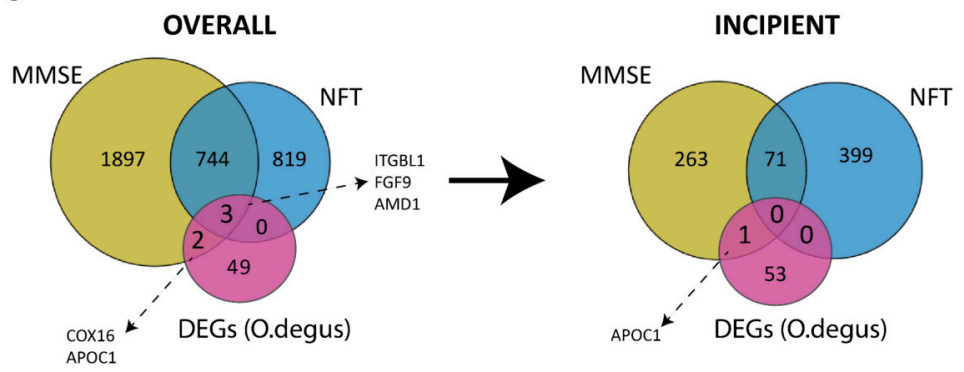

FIGURE 1 | (A) Expression (FPKM values) analysis of 54 differentially expressed genes in PB group compared to GB group with two-way clustering applied. (B) Complete list of KEGG pathways corresponding to 29 up- and 25 down-regulated genes between PB and GB. Columns A and C: KEGG entry for up- and down-regulated genes, Columns B and D: Pathway names for up- and down-regulated genes. (C) Comparison of differentially expressed genes in PB vs. GB and AD-related human genes. Overall AD-correlated genes and AD-correlated genes with incipient AD stage.

in our study (Table S10). The relative change in gene expression levels across AD-like and control samples is shown in Figure 1A.

To determine the function of DEGs, a Gene Ontology (GO) enrichment analysis was performed (Table S7). The up-regulated genes were enriched notably in chromosome related processes, which include chromosome condensation and chromosome organization. GO results showed also that upregulated genes were largely involved in amine biosynthesis, in particular biosynthesis of polyamines. In contrast, most down-regulated genes were mainly engaged in ion homeostasis related processes, such as sodium-independent organic anion transport, hyperosmotic salinity response or regulation of cellular $\mathrm{pH}$ reduction. To fully determine which pathways could be directly affected in $\mathrm{AD}$, DEGs were analyzed using KEGG PATHWAY Database (Braidy et al., 2012). The up-regulated genes were observed to be mainly part of signaling pathways including MAPK, Rap1, Ras and neurotransmission (cholinergic synapse), while down-regulated genes were part of pathways related to $\mathrm{AD}$, Parkinson's disease and Huntington's disease (Figure 1B). Specifically down-regulation of COX8A gene. The COX8A encodes subunit VIIIA of cytochrome $c$ oxidase, a crucial element for the formation of complex IV, which is a part of electron transport chain (ETC) in mitochondria. This is consistent with previously published data, as AD has been already linked to low activity of brain cytochrome c oxidase (Alleyne et al., 2011; Readnower et al., 2011). A substantial number of other down-regulated genes were also found to participate in different metabolic pathways.

\section{Comparison of Human and O. degus AD-like Brain Transcriptomes}

To gain insights to how $O$. degus could be employed as a natural model for studying the pathogenesis of $\mathrm{AD}$, a human $\mathrm{AD}$ brain transcriptome was analyzed using RNA-seq data derived from hippocampus tissue of $\mathrm{AD}$ subjects and controls (Bai et al., 2013). Analysis of DEGs resulted in identification of 1,572 up and 1,391 down-regulated genes, which were further compared to DEGs identified for O. degus (Table S8). This comparison revealed overlap between seven genes, including FGF9, WISP1 and CPNE7 that are known to be linked with AD. Both FGF9 and WISP1 were observed to show the opposite expression changes for human and $O$. degus, while the altered expression of CPNE7 was consistent between both species. Other common 
DEGs included one up-regulated gene: ITGBL1, and two downregulated genes: C1orf95, LRRC55, as well as IL1RAPL2, which was down-regulated in $O$. degus brain and up-regulated in human hippocampus. To further compare human and O. degus AD-like brain transcriptomes, GO enrichment was employed. GO analysis revealed that that up-regulated genes for human were mainly involved in system and tissue development, in particular circulatory system and vasculature development (Table S9). This is to some extend consistent with biological processes described for O. degus up-regulated genes, as majority of enriched terms were biosynthesis related. Down-regulated genes were significantly enriched in neurological processes including signal transduction and neurogenesis. Similar to O. degus, AD affects glutamate secretion and glutamate receptor signaling pathways in human brain. Interestingly, genes involved in regulation of ion transport was also affected, supporting the relationship between cellular stress signals and AD.

\section{Comparison of O. degus DEGs and Human AD-related Genes}

To investigate the potential role of $O$. degus DEGs in human $\mathrm{AD}$ progression, we compared our results to a set of $\mathrm{AD}$-related genes, generated using Affymetrix expression microarrays across different stages of human $\mathrm{AD}$ : incipient, moderate and severe, as well as control samples (Blalock et al., 2011). AD-related genes were defined as genes, whose expression was positively or negatively correlated with results of MiniMental Status Examination (MMSE) and neurofibrillary tangles (NFT) counts across three AD categories (Blalock et al., 2004). Interestingly, five $O$. degus $\mathrm{DEG}$ s could be linked to $\mathrm{AD}$-related genes in all subjects (overall correlation) this included COX16, APOC1, ITGBL1, FGF9, and AMD1. All five DEGs correlated with MMSE scores, while three: ITGBL1, FGF9, and AMD1 were both MMSE and NFT-correlated. However, only $A M D 1$ showed a similar correlation with incipient AD (Figure 1C).

\section{DISCUSSION}

The purpose of the present study was to characterize the brain transcriptome of the $O$. degus to support its use as a natural model of $\mathrm{AD}$, by comparing the transcriptomes of $\mathrm{AD}$-like and healthy $O$. degus. This study is, to the best of our knowledge, the first report of $O$. degus brain whole transcriptome sequencing (RNA-seq). Our results revealed in O. degus a number of genes previously implicated in $\mathrm{AD}$ and related disorders indicating that this model is a suitable alternative to transgenic mice models for $\mathrm{AD}$ research. Because the $\mathrm{AD}$-pathology can vary widely with animal age we carefully matched control and AD-like groups. Animals aged 3 years have optimal AD-like pathology development namely, the presence of beta-amyloid deposits as has been previously reported (Inestrosa et al., 2005; Ardiles et al., 2012; Deacon et al., 2015). This allowed for an acceptable signal dynamic for the experimental analysis. The sample size used for this study was calculated on the magnitude of the biological effect and the inherent variability of the target being measured, in this case the presence or absence of beta-amyloid deposits. We also considered the variability in the behavioral measures, which as previously reported (Deacon et al., 2015) showed clustering in two cohort of animals, AD-like and controls in the tests of daily living.

This work has identified up-regulated genes involved in AD including the CHRNA6 gene, which encodes a sub-type of neuronal nicotinic acetylcholine receptor widely associated to $\mathrm{AD}$, which are mostly linked to $\mathrm{A} \beta$ aggregation theory and that was found previously affected in AD patients (Lombardo and Maskos, 2015). Another DEG AMD1 (which encodes an intermediate enzyme relevant to the polyamine biosynthesis) has an altered activity in $\mathrm{AD}$ and has also been implicated in schizophrenia and mood disorders, and was found up-regulated in affected AD-like O. degus (Morrison et al., 1993; Fiori and Turecki, 2008). The down-regulated genes found in the brains of AD-like $O$. degus included WNT1 inducible signaling pathway protein 1 (WISP1) (Varela-Nallar and Inestrosa, 2013). This gene previously implicated in neurodegeneration by regulation of mitochondrial signaling and apoptosis is a downstream target in the Wnt1 signaling pathway (Wang et al., 2012). Wnt signaling that involves Wnt1 and WISP1 is becoming recognized as a vital neuroprotective component during $\mathrm{AD}$. WISP1 stops p53 mediated DNA damage and apoptosis offering neuronal protection by blocking cytochrome c release ( $\mathrm{Su}$ et al., 2008; Wang et al., 2012). Mitochondrial-related genes were also found to be differentially expressed in AD-like O. degus brains, including the Cytochrome C Oxidase Subunit 8A (COX8A) that has been associated with the reduction of neuronal energy metabolism and mitochondrial dysfunction observed in $\mathrm{AD}$ patients by the inhibition of COX activity as a result of binding to $A \beta$-subunits (Lustbader et al., 2004; Liang et al., 2008).

The comparison of human and $O$. degus AD-like brain transcriptomes, revealed seven common genes that were deregulated. This included fibroblast growth factor 9 (FGF9), WISP1 and Copine VII (CPNE7). Interestingly, the FGF9 gene is known to be related to the MAPK pathway affected in AD patients (Antonell et al., 2013), and is one of the therapeutic targets currently used in AD treatment (Zhang et al., 2014).

Retinal abnormalities have also been reported to be associated to $\mathrm{AD}$ (Berisha et al., 2007). O. degus naturally develops cataracts, an eye dysfunction linked to neurodegeneration (Inestrosa et al., 2005, 2015; Braidy et al., 2015; Szabadfi et al., 2015). Our study revealed deregulated expression of two genes: FRMD7 and ADAMTS9, which were previously associated to retinal abnormalities. The FRMD7 gene plays role in retinal and neurite development, as well as in the neuronal outgrowth. It was also connected to X-linked infantile nystagmus, a disease characterized by abnormal eye function (BettsHenderson et al., 2010). The ADAMTS9 gene is also known to be involved in abnormal eye development. Extracellular matrix is a key mediator in the pathogenesis of age related macular degeneration and includes ADAMTS9. ADAMTS9 activates $A K T$ promoting photoreceptor degeneration and consequently membrane thickening and damage of retinal pigmentary cells. Moreover regulation of mTOR by ADAMTS9 leads to angiogenesis via VEGF $A$ and TSP-1 this way regulating angiogenesis in the eye (Wight, 2005; Parry et al., 2009). Moreover, the activity disorders of ADAMTS9 regulation of the AKT/mTOR pathway leads to glycolysis and glucose 
uptake by increasing hypoxia-inducible factor (HIF)-1 $\alpha$ (HIF1A) also been linked to AD (Avramovich-Tirosh et al., 2010). In humans, an ADAMTS9 gene variant is associated with type 2 diabetes (T2DM) is most frequently linked with aging, cognitive impairment, $\mathrm{AD}$-associated neuronal APP-A $\beta$ deposits (de la Monte and Wands, 2005). In relation to these results, the O. degus has insulin resistance and naturally develops type 2 diabetes and associated cataracts when fed with a diet high in glucose (Datiles and Fukui, 1989).

We have also identified APOC-I (apolipoprotein C-I) to be deregulated in $\mathrm{AD}$-like $\mathrm{O}$. degus brain samples. Notably, APOC-I is part of the APOE/C-I/C-IV/C-II gene cluster on the chromosome 19 (Smit et al., 1988) and APOE modulates its expression. This gene encodes a small apolipoprotein that is associated with A $\beta$ plaques (Kamino et al., 1996). APOC-I plays a critical role in CNS homeostasis, since altered expression impairs memory (Abildayeva et al., 2008). Moreover, is to be highlighted that APOC-I co-localizes with $\mathrm{A} \beta$ plaques in the brain in $\mathrm{AD}$ (Abildayeva et al., 2008).

The pathogenic mechanism resulting in the onset and progression of neurodegenerative diseases like $\mathrm{AD}$ is often associated with genetic variants and mutations and interactions with environmental impacts, lifestyle risk factors, and slowly evolving molecular changes due to aging (Jicha and Carr, 2010; Duchen, 2012). Thus, a number of AD research avenues could be investigated in this model other than RNA analysis. For example post-translationally through modification of protein residues like phosphorylation.

In this study we focused in whole brain, so studies of additional brain regions are needed and may point to different genes and additional pathways. The RNA-seq uncovered multiple DE transcripts, several of these are novel and some have been previously implicated in AD like WISP1 and APOC1. Being the present work a pilot study we have probably uncovered only a fraction of what these data may reveal about this model and its link with AD supporting further work with this species. Future work is underway in our laboratory to elucidate the characterization of global gene expression of young and old $O$. degus by analysing tissue from different brain areas. To our knowledge this is the first $O$. degus brain transcriptome study. Salazar et al. (2016), compare the sequences of genes in relation to $\mathrm{AD}$ using published data from $O$. degus, human and other rodents. They analyzed tau, APP, Apolipoprotein E (APOE), Presenilin 1 (Psen 1 ), and $A \beta$-peptide sequences in correlation with specific human variants associated with $\mathrm{AD}$. Their findings revealed that $O$. degus have the arginine substitution present in the ApoE4 pathogenic human allele; Psen1 gene showed a greater relatedness between the isoforms of human, degus, guinea pig, and mole rat, compared to those of others rodents such the rat, and also described that $O$. degus $\mathrm{A} \beta$-peptide sequence presents high homology with the human protein, differing in only one amino acid (Salazar et al., 2016). Furthermore, Deacon et al. (2015) shows that AD-like O. degus have high levels of A $\beta$-peptide aggregates, APP, TNF-a, IL-6, IFN-a, and the oxidative stress marker NFE2L2 when compared to healthy control $O$. degus, suggesting that $\mathrm{AD}$-like $O$. degus present increased inflammation as is observed in human $\mathrm{AD}$ patients
(Deacon et al., 2015). Overall the study of others and the present explorative transcriptome analysis revealed that the $\mathrm{AD}$ like $O$. degus shares common affected genes with those have been implicated in human $\mathrm{AD}$ and therefore provides further characterization of the $O$. degus as a relevant natural model. $O$. degus shows a correlation of expression with human ADrelated genes making this model a powerful preclinical tool to characterize target effects of novel therapeutics in AD. This study provides strong evidence to support the $O$. degus as a valuable natural model for preclinical work in $\mathrm{AD}$ research.

\section{ETHICS STATEMENT}

This study was carried out under the approval of the ethics committee of the Faculty of Sciences, Universidad de Chile, directed by Dr. Marco Méndez and integrated by:-Dr. Eduardo Friedman-Dr. Victoria Guixé-Dr. Madeleine LamborotDr. Roberto Morales-Dr. Aurelio San Martín-Dr. Cecilia Vergara.

\section{DATA AVAILABILITY}

All the Octodon degus transcriptome data is available through the NCBI using accession PRJNA326273.

\section{AUTHOR CONTRIBUTIONS}

FA and BU contributed to the experimental procedures, data analysis and the manuscript preparation. FC, AV, EP, SN, and DL contributed to created the bioinformatic pipeline for sequencing data analysis. LF contributed to the experimental procedures and data analysis. RMJD, MH, and RD contributed to comprehensive analysis of the results and to the manuscript preparation. RV, $\mathrm{RG}$, and PC contributed to the funding of the project, the experimental design and the manuscript preparation.

\section{ACKNOWLEDGMENTS}

This research was funded by GeN.DDI Ltd (London, UK) and by the Chilean National Commission for Scientific and Technological Research Grants 1090794, 1140548, ICM-P05002 and PFB-23-CONICYT (to the Institute of Ecology and Biodiversity). Research was conducted under permit No. 5193 issued by the Servicio Agrícola y Ganadero, Chile, with the supervision of the Ethics Committee of the Faculty of Sciences, Universidad de Chile. We acknowledge support of the Spanish Ministry of Economy and Competitiveness, "Centro de Excelencia Severo Ochoa 2013-2017," SEV-2012-0208 and Project PT13/0001/0021 (ISCIII - Subdirección General de Evaluación y Fomento de la Investigación/FEDER “Una Manera de hacer Europa").

\section{SUPPLEMENTARY MATERIAL}

The Supplementary Material for this article can be found online at: http://journal.frontiersin.org/article/10.3389/fnagi. 2017.00064/full\#supplementary-material 


\section{REFERENCES}

Abildayeva, K., Berbée, J. F. P., Blokland, A., Jansen, P. J., Hoek, F. J., Meijer, O., et al. (2008). Human apolipoprotein C-I expression in mice impairs learning and memory functions. J. Lipid Res. 49, 856-869. doi: 10.1194/jlr.M700518-JLR200

Alleyne, T., Mohan, N., Joseph, J., and Adogwa, A. (2011). Unraveling the role of metal ions and low catalytic activity of cytochrome C oxidase in Alzheimer's disease. J. Mol. Neurosci. 43, 284-289. doi: 10.1007/s12031-010-9436-8

Altschul, S. (1997). Gapped BLAST and PSI-BLAST: a new generation of protein database search programs. Nucleic Acids Res. 25, 3389-3402. doi: 10.1093/nar/25.17.3389

Antonell, A., Lladó, A., Altirriba, J., Botta-Orfila, T., Balasa, M., Fernández, M., et al. (2013). A preliminary study of the whole-genome expression profile of sporadic and monogenic early-onset Alzheimer's disease. Neurobiol. Aging 34, 1772-1778. doi: 10.1016/j.neurobiolaging.2012.12.026

Ardiles, A. O., Tapia-Rojas, C. C., Mandal, M., Alexandre, F., Kirkwood, A., Inestrosa, N. C., et al. (2012). Postsynaptic dysfunction is associated with spatial and object recognition memory loss in a natural model of Alzheimer's disease. Proc. Natl. Acad. Sci. U.S.A. 109, 13835-13840. doi: 10.1073/pnas.1201209109

Avramovich-Tirosh, Y., Bar-Am, O., Amit, T., Youdim, M. B. H., and Weinreb, O. (2010). Up-regulation of hypoxia-inducible factor (HIF)- $1 \alpha$ and HIF-target genes in cortical neurons by the novel multifunctional iron chelator anti-Alzheimer drug, M30. Curr. Alzheimer Res. 7, 300-306. doi: 10.2174/156720510791162403

Bai, B., Hales, C. M., Chen, P.-C., Gozal, Y., Dammer, E. B., Fritz, J. J., et al. (2013). U1 small nuclear ribonucleoprotein complex and RNA splicing alterations in Alzheimer's disease. Proc. Natl. Acad. Sci. U.S.A. 110, 16562-16567. doi: $10.1073 /$ pnas. 1310249110

Berisha, F., Feke, G. T., Trempe, C. L., McMeel, J. W., and Schepens, C. L. (2007). Retinal abnormalities in early alzheimer's disease. investig opthalmology Vis. Sci. 48, 2285. doi: 10.1167/iovs.06-1029

Betts-Henderson, J., Bartesaghi, S., Crosier, M., Lindsay, S., Chen, H.-L., Salomoni, P., et al. (2010). The nystagmus-associated FRMD7 gene regulates neuronal outgrowth and development. Hum. Mol. Genet. 19, 342-351. doi: $10.1093 / \mathrm{hmg} / \mathrm{ddp} 500$

Birch, A. M., Katsouri, L., and Sastre, M. (2014). Modulation of inflammation in transgenic models of Alzheimer's disease. J. Neuroinflam. 11:25. doi: 10.1186/1742-2094-11-25

Blalock, E. M., Buechel, H. M., Popovic, J., Geddes, J. W., and Landfield, P. W. (2011). Microarray analyses of laser-captured hippocampus reveal distinct gray and white matter signatures associated with incipient Alzheimer's disease. J. Chem. Neuroanat. 42, 118-126. doi: 10.1016/j.jchemneu.2011.06.007

Blalock, E. M., Geddes, J. W., Chen, K. C., Porter, N. M., Markesbery, W. R., and Landfield, P. W. (2004). Incipient Alzheimer's disease: microarray correlation analyses reveal major transcriptional and tumor suppressor responses. Proc. Natl. Acad. Sci. U.S.A. 101, 2173-2178. doi: 10.1073/pnas.0308512100

Blasko, I., Stampfer-Kountchev, M., Robatscher, P., Veerhuis, R., Eikelenboom, P., and Grubeck-Loebenstein, B. (2004). How chronic inflammation can affect the brain and support the development of Alzheimer's disease in old age: the role of microglia and astrocytes. Aging Cell. 3, 169-176. doi: 10.1111/j.1474-9728.2004.00101.x

Braidy, N., Muñoz, P., Palacios, A. G., Castellano-Gonzalez, G., Inestrosa, N. C., Chung, R. S., et al. (2012). Recent rodent models for Alzheimer's disease: clinical implications and basic research. J. Neural Transm. 119, 173-195. doi: 10.1007/s00702-011-0731-5

Braidy, N., Poljak, A., Jayasena, T., Mansour, H., Inestrosa, N. C., and Sachdev, P. S. (2015). Accelerating Alzheimer's research through "natural"animal models. Curr. Opin. Psychiatry 28, 155-164. doi: 10.1097/YCO.0000000000000137

Castellani, R. J., and Perry, G. (2012). Pathogenesis and disease-modifying therapy in Alzheimer's disease: the flat line of progress. Arch. Med. Res. 43, 694-698. doi: 10.1016/j.arcmed.2012.09.009

Correa, L. A., León, C., Ramírez-Estrada, J., Soto-Gamboa, M., Sepúlveda, R. D., and Ebensperger, L. A. (2016). Masculinized females produce heavier offspring in a group living rodent. J. Anim. Ecol. 85, 1552-1562. doi: 10.1111/1365-2656.12588

Datiles, M. B. III, and Fukui, H. (1989). Cataract prevention in diabetic Octodon degus with Pfizer's sorbinil. Curr. Eye Res. 8, 233-237.
Deacon, R. M. J. (2014). A novel approach to discovering treatments for alzheimer's disease. J. Alzheimer Dis. Park. 4:142. doi: 10.4172/2161-0460.1000142

Deacon, R. M. J. (2006). Burrowing in rodents: a sensitive method for detecting behavioral dysfunction. Nat. Protoc. 1, 118-121. doi: 10.1038/nprot.2006.19

Deacon, R. M. J., Altimiras, F. J., Bazan-Leon, E. A., Pyarasani, R. D., Nachtigall, F. M., Santos, L. S., et al. (2015). Natural AD-Like neuropathology in Octodon degus: impaired burrowing and neuroinflammation. Curr. Alzheimer Res. 12, 314-322. doi: 10.2174/1567205012666150324181652

de la Monte, S. M., and Wands, J. R. (2005). Review of insulin and insulin-like growth factor expression, signaling, and malfunction in the central nervous system: relevance to Alzheimer's disease. J. Alzheimer's Dis. 7, 45-61. Available online at: http://content.iospress.com/articles/journal-of-alzheimers-disease/ jad00401

Dobin, A., Davis, C. A., Schlesinger, F., Drenkow, J., Zaleski, C., Jha, S., et al. (2013). STAR: ultrafast universal RNA-seq aligner. Bioinformatics 29, 15-21. doi: 10.1093/bioinformatics/bts635

Duchen, M. R. (2012). Mitochondria, calcium-dependent neuronal death and neurodegenerative disease. Pflügers Arch. 464. 111-121. doi: 10.1007/s00424-012-1112-0

Ebensperger, L., and Hurtado, M. J. (2005). Seasonal changes in the time budget of degus, Octodon degus. Behaviour 142, 91-112. doi: 10.1163/1568539053627703

Fiori, L. M., and Turecki, G. (2008). Implication of the polyamine system in mental disorders. J. Psychiatry Neurosci. 33, 102-110. Available online at: http://jpn.ca/ vol33-issue2/33-2-102/

Götz, S., García-Gómez, J. M., Terol, J., Williams, T. D., Nagaraj, S. H., Nueda, M. J., et al. (2008). High-throughput functional annotation and data mining with the Blast2GO suite. Nucleic Acids Res. 36, 3420-3435. doi: 10.1093/nar/gkn176

Guigo, R., Dermitzakis, E. T., Agarwal, P., Ponting, C. P., Parra, G., Reymond, A., et al. (2003). Comparison of mouse and human genomes followed by experimental verification yields an estimated 1,019 additional genes. Proc. Natl. Acad. Sci. U.S.A. 100, 1140-1145. doi: 10.1073/pnas.0337561100

Guigó, R., Knudsen, S., Drake, N., and Smith, T. (1992). Prediction of gene structure. J. Mol. Biol. 226, 141-157. doi: 10.1016/0022-2836(92)90130-C

Haas, B. J. (2003). Improving the Arabidopsis genome annotation using maximal transcript alignment assemblies. Nucleic Acids Res. 31, 5654-5666. doi: $10.1093 /$ nar/gkg770

Haas, B. J., Salzberg, S. L., Zhu, W., Pertea, M., Allen, J. E., Orvis, J., et al. (2008). Automated eukaryotic gene structure annotation using Evidencemodeler and the program to assemble spliced alignments. Genome Biol. 9:R7. doi: $10.1186 / \mathrm{gb}-2008-9-1-\mathrm{r} 7$

Harrow, J., Frankish, A., Gonzalez, J. M., Tapanari, E., Diekhans, M., Kokocinski, F., et al. (2012). GENCODE: the reference human genome annotation for The ENCODE Project. Genome Res. 22, 1760-1774. doi: 10.1101/gr.1353 50.111

Inestrosa, N. C., Reyes, A. E., Chacón, M. A., Cerpa, W., Villalón, A., Montiel, J., et al. (2005). Human-like rodent amyloid-beta-peptide determines Alzheimer pathology in aged wild-type Octodon degu. Neurobiol. Aging 26, 1023-1028. doi: 10.1016/j.neurobiolaging.2004.09.016

Inestrosa, N. C., Ríos, J. A., Cisternas, P., Tapia-Rojas, C., Rivera, D. S., Braidy, N., et al. (2015). Age progression of neuropathological markers in the brain of the chilean rodent Octodon degus, a natural model of Alzheimer's disease. Brain Pathol. 25, 679-691. doi: 10.1111/bpa.12226

Iwata, H., and Gotoh, O. (2012). Benchmarking spliced alignment programs including Spaln2, an extended version of Spaln that incorporates additional species-specific features. Nucleic Acids Res. 40, e161. doi: 10.1093/nar/gks708

Jicha, G. A., and Carr, S. A. (2010). Conceptual evolution in Alzheimer's disease: implications for understanding the clinical phenotype of progressive neurodegenerative disease. J. Alzheimer's Dis. 19, 253-272. doi: 10.3233/JAD-2010-1237

Kamino, K., Yoshiiwa, A., Nishiwaki, Y., Nagano, K., Yamamoto, H., Kobayashi, T., et al. (1996). Genetic association study between senile dementia of Alzheimer's Type and APOE/C1/C2 gene cluster. Gerontology 42(Suppl. 1), 12-19. doi: 10.1159/000213820

Kanehisa, M., Goto, S., Sato, Y., Furumichi, M., and Tanabe, M. (2012). KEGG for integration and interpretation of large-scale molecular data sets. Nucleic Acids Res. 40(Database issue), D109-D114. doi: 10.1093/nar/gkr988

Kanehisa, M., Goto, S., Sato, Y., Kawashima, M., Furumichi, M., and Tanabe, M. (2014). Data, information, knowledge and principle: back to 
metabolism in KEGG. Nucleic Acids Res. 42(Database issue), D199-D205. doi: 10.1093/nar/gkt1076

Laurijssens, B., Aujard, F., and Rahman, A. (2013). Animal models of Alzheimer's disease and drug development. Drug Discov. Today Technol. 10, e319-e327. doi: 10.1016/j.ddtec.2012.04.001

Li, B., and Dewey, C. N. (2011). RSEM: accurate transcript quantification from RNA-Seq data with or without a reference genome. BMC Bioinformatics 12:323. doi: 10.1186/1471-2105-12-323

Li, H., Handsaker, B., Wysoker, A., Fennell, T., Ruan, J., Homer, N., et al. (2009). The sequence alignment/map format and SAMtools. Bioinformatics. 25, 2078-2079. doi: 10.1093/bioinformatics/btp352

Liang, W. S., Reiman, E. M., Valla, J., Dunckley, T., Beach, T. G., Grover, A., et al. (2008). Alzheimer's disease is associated with reduced expression of energy metabolism genes in posterior cingulate neurons. Proc. Natl. Acad. Sci. U.S.A. 105, 4441-4446. doi: 10.1073/pnas.0709259105

Lombardo, S., and Maskos, U. (2015). Role of the nicotinic acetylcholine receptor in Alzheimer's disease pathology and treatment. Neuropharmacology 96, 255-262. doi: 10.1016/j.neuropharm.2014.11.018

Lustbader, J. W., Cirilli, M., Lin, C., Xu, H. W., Takuma, K., Wang, N., et al. (2004). ABAD directly links Abeta to mitochondrial toxicity in Alzheimer's disease. Science 304, 448-452. doi: 10.1126/science.1091230

Mangialasche, F., Solomon, A., Winblad, B., Mecocci, P., and Kivipelto, M. (2010). Alzheimer's disease: clinical trials and drug development. Lancet Neurol. 9, 702-716. doi: 10.1016/S1474-4422(10)70119-8

Moriya, Y., Itoh, M., Okuda, S., Yoshizawa, A. C., and Kanehisa, M. (2007). KAAS: an automatic genome annotation and pathway reconstruction server. Nucleic Acids Res. 35, W182-W185. doi: 10.1093/nar/ gkm321

Morrison, L. D., Bergeron, C., and Kish, S. J. (1993). Brain S-adenosylmethionine decarboxylase activity is increased in Alzheimer's disease. Neurosci Lett. 154, 141-144. doi: 10.1016/0304-3940(93)90191-M

Mortazavi, A., Williams, B. A., McCue, K., Schaeffer, L., and Wold, B. (2008). Mapping and quantifying mammalian transcriptomes by RNA-Seq. Nat Methods 5, 621-628. doi: 10.1038/nmeth.1226

Nazem, A., Sankowski, R., Bacher, M., and Al-Abed, Y. (2015). Rodent models of neuroinflammation for Alzheimer's disease. J. Neuroinflam. 12, 74. doi: 10.1186/s12974-015-0291-y

Parra, G., Agarwal, P., Abril, J. F., Wiehe, T., Fickett, J. W., and Guigó, R. (2003). Comparative gene prediction in human and mouse. Genome Res. 13, 108-117. doi: $10.1101 /$ gr.871403

Parry, D. A., Toomes, C., Bida, L., Danciger, M., Towns, K. V., McKibbin, M., et al. (2009). Loss of the metalloprotease ADAM9 leads to cone-rod dystrophy in humans and retinal degeneration in mice. Am. J. Hum. Genet. 84, 683-691. doi: 10.1016/j.ajhg.2009.04.005

Readnower, R. D., Sauerbeck, A. D., and Sullivan, P. G. (2011). Mitochondria, Amyloid $\beta$, and Alzheimer's disease. Int. J. Alzheimers Dis. 2011, 1-5. doi: 10.4061/2011/104545

Robinson, M. D., McCarthy, D. J., and Smyth, G. K. (2010). edgeR: a Bioconductor package for differential expression analysis of digital gene expression data. Bioinformatics. 26, 139-140. doi: 10.1093/bioinformatics/btp616

Rumble, S. M., Lacroute, P., Dalca, A. V., Fiume, M., Sidow, A., and Brudno, M. (2009). SHRiMP: accurate mapping of short color-space reads. PLoS Comput. Biol. 5:e1000386. doi: 10.1371/journal.pcbi.1000386

Salazar, C., Valdivia, G., Ardiles, Á. O., Ewer, J., and Palacios, A. G. (2016). Genetic variants associated with neurodegenerative Alzheimer disease in natural models. Biol. Res. 49, 14. doi: 10.1186/s40659-0160072-9

Saraceno, C., Musardo, S., Marcello, E., Pelucchi, S., and Luca, M. D. (2013). Modeling Alzheimer's disease: from past to future. Front. Pharmacol. 4:77. doi: 10.3389/fphar.2013.00077
Slater, G. S. C., and Birney, E. (2005). Automated generation of heuristics for biological sequence comparison. BMC Bioinformatics. 6:31. doi: 10.1186/1471-2105-6-31

Smit, M., van der Kooij-Meijs, E., Frants, R. R., Havekes, L., and Klasen, E. C. (1988). Apolipoprotein gene cluster on chromosome 19. Hum. Genet. 78, 90-93.

Stanke, M., Keller, O., Gunduz, I., Hayes, A., Waack, S., and Morgenstern, B. (2006a). AUGUSTUS: ab initio prediction of alternative transcripts. Nucleic Acids Res. 34(Web Server issue), W435-W439. doi: 10.1093/nar/gkl200

Stanke, M., Schöffmann, O., Morgenstern, B., and Waack, S. (2006b). Gene prediction in eukaryotes with a generalized hidden Markov model that uses hints from external sources. BMC Bioinformatics 7:62. doi: 10.1186/1471-2105-7-62

Su, Y. A., Wu, J., Zhang, L., Zhang, Q., Su, D. M., He, P., et al. (2008). Dysregulated mitochondrial genes and networks with drug targets in postmortem brain of patients with posttraumatic stress disorder (PTSD) revealed by human mitochondria-focused cDNA microarrays. Int. J. Biol. Sci. 4, 223-235. doi: $10.7150 /$ ijbs. 4.223

Szabadfi, K., Estrada, C., Fernandez-Villalba, E., Tarragon, E., Setalo, G., Izura, V., et al. (2015). Retinal aging in the diurnal Chilean rodent (Octodon degus): histological, ultrastructural and neurochemical alterations of the vertical information processing pathway. Front. Cell Neurosci. 9:126. doi: 10.3389/fncel.2015.00126

Tarragon, E., Lopez, D., Estrada, C., Ana, G.-C., Schenker, E., Pifferi, F., et al. (2013). Octodon degus: a model for the cognitive impairment associated with Alzheimaer's disease. CNS Neurosci Ther. 19, 643-648. doi: 10.1111/cns.12125

Varela-Nallar, L., and Inestrosa, N. C. (2013). Wnt signaling in the regulation of adult hippocampal neurogenesis. Front. Cell Neurosci. 7:100. doi: 10.3389/fncel.2013.00100

Wang, S., Chong, Z. Z., Shang, Y. C., and Maiese, K. (2012). Wnt1 inducible signaling pathway protein 1 (WISP1) blocks neurodegeneration through phosphoinositide 3 kinase/Akt1 and apoptotic mitochondrial signaling involving Bad, Bax, Bim, and Bcl-xL. Curr. Neurovasc. Res. 9, 20-31. doi: $10.2174 / 156720212799297137$

Wight, T. N. (2005). The ADAMTS proteases, extracellular matrix, and vascular disease: waking the sleeping giant(s)! Arterioscler. Thromb. Vasc. Biol. 25, 12-14. doi: 10.1161/01.ATV.0000150043.43083.aa

Windisch, M. (2014). We can treat Alzheimer's disease successfully in mice but not in men: failure in translation? A perspective. Neurodegener Dis. 13, 147-150. doi: 10.1159/000357568

Zdobnov, E. M., and Apweiler, R. (2001). InterProScan-an integration platform for the signature-recognition methods in InterPro. Bioinformatics 17, 847-848. doi: 10.1093/bioinformatics/17.9.847

Zhang, C., Chen, J., Feng, C., Shao, X., Liu, Q., Zhang, Q., et al. (2014). Intranasal nanoparticles of basic fibroblast growth factor for brain delivery to treat Alzheimer's disease. Int. J. Pharm. 461, 192-202. doi: 10.1016/j.ijpharm.2013.11.049

Conflict of Interest Statement: The authors declare that the research was conducted in the absence of any commercial or financial relationships that could be construed as a potential conflict of interest.

Copyright (C) 2017 Altimiras, Uszczynska-Ratajczak, Camara, Vlasova, Palumbo, Newhouse, Deacon, Farias, Hurley, Loyola, Vásquez, Dobson, Guigó and Cogram. This is an open-access article distributed under the terms of the Creative Commons Attribution License (CC BY). The use, distribution or reproduction in other forums is permitted, provided the original author(s) or licensor are credited and that the original publication in this journal is cited, in accordance with accepted academic practice. No use, distribution or reproduction is permitted which does not comply with these terms. 